\title{
ESTUDIO DEL PROCESO DE FOTODEGRADACION DE $\beta$ - CAROTENO EN UNA SOLUCION DE TETRAHIDROFURANO (THF) USANDO ESPECTROSCOPIA DE ABSORCION OPTICA Y FLUORESCENCIA
}

\author{
STUDY OF THE PHOTODEGRADATION PROCESS OF $\beta$-CAROTENE IN \\ THF SOLUTION USING OPTICAL ABSORPTION AND FLUORESCENCE \\ SPECTROSCOPY
}

'José A. Tiburcio Moreno, ${ }^{2,3}$ Florencio E. Hernández

\begin{abstract}
RESUMEN
En este trabajo, presentamos el estudio espectroscópico de la fotodegradación ultravioleta de $\beta$-caroteno en una solución de THF a dos diferentes longitudes de onda. El análisis temporal del proceso, bajo una irradiación a $366 \mathrm{~nm}$ y $254 \mathrm{~nm}$, muestra una dinámica rápida a longitudes de anda cortas. Asimismo, se observa una cinética de primer orden en ambas longitudes de onda lo que confirma la descomposición unimolecular del modelo propuesto en la literatura. Este trabajo se estima a ser de relevanciá en la descomposición fotoinducida para el estudio y empleo de esta molécula en la industria de antioxidantes naturales, al mentos, drogas y farmacéutica.
\end{abstract}

Palabras clave: fotodegradación, descomposición fotoinducida.

ABSTRACT

Herein, we present the spectroscopic study of the UV photo-degradation of $\beta$-carotene in THF solution at two different wavelengths The temporal analysis of the process, upon irradiation at $366 \mathrm{~nm}$ and $254 \mathrm{~nm}$, shows a faster dynamics at shorter wavelength. The observed first-order kinetic at both wavelengths confirm the unimolecular decomposition model proposed in the literature. This work is expected to be of great relevance for the study photo-induced decomposition of natural antioxidants in the food, drug, and pharmaceutical industry

Keywords: fotodegradación, descomposición fotoinducida.

\section{INTRODUCCIÓN}

Los carotenoides constituyen una clase importante de moléculas que exhiben un alto grado de deslocalización electrónica y dinámica ultra rápida (Vivas et al., 2011). El $\beta$ caroteno es uno de los miembros de la familia de los caretenoides más estudiado debido a su rol importante en procesos fotobiológicos, fotosintesis y fotoprotccción (Siefermann-Harms, 1985). Este compuesto es encontrado principalmente en vegetales que el cuerpo humano puede convertir en vitamina $\mathrm{A}$, la cual actúa como un antioxidante. La forma natural del $\beta$-caroteno existe en dos isomeros; alltrans- $\beta$-caroteno y 9-cis- $\beta$-caroteno. Este ultima presenta una estructura altamente ordenada con alta coherencia y pequeñas propiedades oscilantes en los enlaces C-C (Guan-Nan $t$ t al., 2011). Este isomero puede ser sintetizado para ser vendi- do comercialmente como suplemento alimenticio en la dieta diaria. La molécula all-trans- $\beta$-caroteno es un carotenoide no polar con once enlaces dobles conjugados. Esta molécula presenta una alta actividad antioxidante a través del bloqueo de oxigeno singlete y también como un reactivo con radicales del tipo fenoxil (Baranska et al., 2006). La molécula de $\beta$ caroteno es tambićn el precursor más efectivo de la vitamina A y ha sido reportado en la prevención de algunos tipos de cáncer (Steinmetz \& Potter, 1996) y enfermedades cardiovasculares (Gaziano et al., 1992). En la literatura se reporta que la estabilidad del $\beta$-caroteno se ve comprometida por la exposición a la luz, altas temperaturas y procesos térmicos (Bonnie \& Choo, 1999). Los carotenoides son degradados como resultado de complejas reacciones de antioxidación las cuales son relacionadas con frecuencia a una degradación oxidativa. Un factor importante en la degradación de los 
Tiburcio, J. \& Hernández, F. Estudio del proceso de fotodegradación de ß-caroteno en una solución de tetrahidrofurano (IHF) usando espectroscopia de absorción óptica y fluorescencia.

carotenoides y en la iniciación de cambios oxidativos es la luz. La degradación fotoinducida de los carotenoides ha sido tema de muchas investigaciones (Carnevale et al., 1980)(Carnevale et al., 1979). Sin embargo, los cambios en la molécula de $\beta$-caroteno todavía no han sido bien comprendidos. El objetivo principal de esta investigación ha sido evaluar estos cambios en la respuesta óptica del $\beta$-caroteno bajo irradiación con luz ultravioleta a dos diferentes longitudes de onda, $254 \mathrm{~nm}$ and $366 \mathrm{~nm}$.

\section{MÉTODOS Y MATERIALES}

\section{Materiales}

La molécula de $\beta$-carotene Type I y tetrahidrofurano (THF) fueron adquiridos de Sigma-Aldrich.

\section{Preparación de las muestras}

Una solución principal fue preparada disolviendo 20 $\mathrm{mg}$ de $\beta$-caroteno en $5 \mathrm{~mL}$ de THF (concentración de la muestra $4 \mathrm{mg} / \mathrm{mL}$ ). De esta solución principal, se prepararon dos nuevas soluciones a las concentraciones de $3,010^{-5} \mathrm{M}$ y $1,510^{6} \mathrm{M}$, las muestras fueron almacenadas en frascos ámbar a una temperatura de $-20^{\circ} \mathrm{C}$.

\section{Irradiación de las muestras}

Las muestras fueron colocadas en una cubeta de cuarzo de $1 \mathrm{~cm}$ de camino óptico, y selladas con parafilm para prevenir la evaporación. La irradiación ultravioleta fue llevada a cabo en periodos de 3 minutos hasta un máximo de 60 minutos, usando una lámpara de alta intensidad ultravioleta; modelo ENF-260C (Spectronic Corporation, Westbury, New York, USA) a $350 \mathrm{~W} / \mathrm{cm}^{2}$ para $254 \mathrm{~nm}$ y 390 $\mathrm{W} / \mathrm{cm}^{2}$ para $366 \mathrm{~nm}$

\section{Medidas de los espectros de absorbancia}

Los espectros de absorción lineal fueron medidos usando un espectrofotómetro de haz simple (Agilent 8453 Diode Array UV-Vis), desde $350 \mathrm{~nm}$ a $540 \mathrm{~nm}$, en una celda de cuarzo de $1 \mathrm{~cm}$ de camino óptico a una concentración de $3,010^{-5} \mathrm{M}$. las contribuciones del solvente y de la celda de

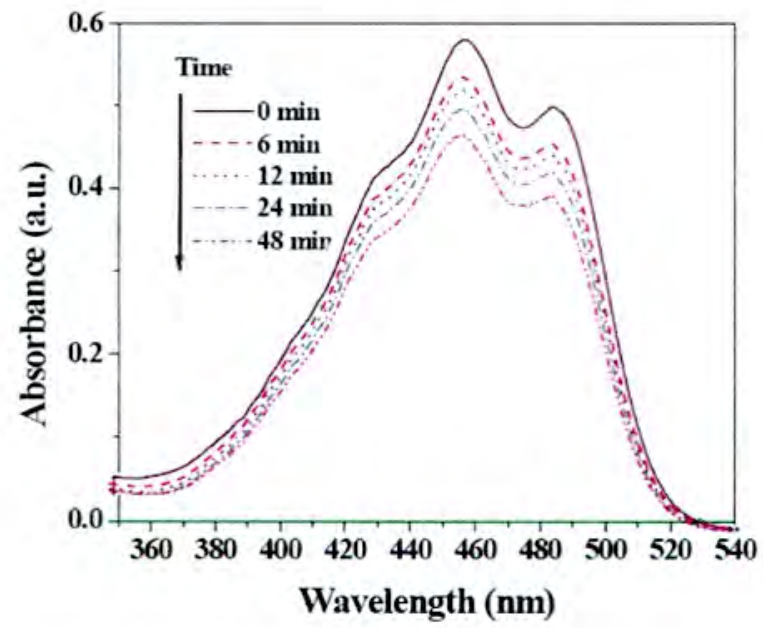

1 (a) cuarzo no fueron consideradas en todos los espectros.

\section{Medidas de los espectros de fluorescencia}

Los espectros de fluorescencia fueron determinados con un espectrómetro USB2000 Fiber Optic Spectrometer (Ocean Optics, Inc.). Los espectros de emisión fueron obtenidos bajo un arreglo perpendicular con un diodo láser de intensidad $10 \mathrm{~mW} / \mathrm{cm}^{2}$ y una longitud de onda de $410 \mathrm{~nm}$. Fueron analizadas dos diferentes soluciones a las concentraciones de $3,010^{-5} \mathrm{My} 1,510^{6} \mathrm{M}$.

\section{RESULTADOS Y DISCUSIÓN}

En la figura 1(a) se muestra el espectro de absorción de la solución de $\beta$-carotene/THF $\left(3.010^{-5} \mathrm{M}\right)$ a través de 48 min de exposición a la irradiación UV a longitud de onda de $366 \mathrm{~nm}$. En primer lugar, debemos notar que en todos los espectros, registrados entre $350 \mathrm{~nm}$ hasta $540 \mathrm{~nm}$, presentan los tres picos de absorción característicos de esta molécula, es decir dos picos principales centrados en $455 \mathrm{~nm}$ y $485 \mathrm{~nm}$, y una pequeña meseta situada en $435 \mathrm{~nm}$ (Krisko, 2004). Luego, se observa que después de 48 min de irradiación los espectros de absorción muestran un cambio moderado en la absorbancia en aproximadamente $20 \%$. Este resultado sugiere que la molécula de $\beta$-caroteno no sufre una fotodescomposición significante a esta longitud de onda. Estos resultados están en acuerdo con resultados previamente reportados en Sorg et al. (1999) y Allwood \& Plane (1984).

En la figura 1(b), presentamos los espectros de absorción de una solución similar de $\beta$-caroteno/THF después de ser irradiada a $254 \mathrm{~nm}$ por un periodo de 24 minutos. En este proceso los tres picos principales de absorción decrecen sustancialmente con el tiempo de irradiación. Se puede observar que después de solo $20 \mathrm{~min}$ de irradiación los espectros de absorbancia decrecen en amplitud en aproximadamente $90 \%$. Esta rápida cinética del proceso observado a cortas longitudes de onda es atribuida a la más eficiente fotodegradación de la molécula de $\beta$-caroteno la cual toma lugar casi exclusivamente a través de sus estados single-

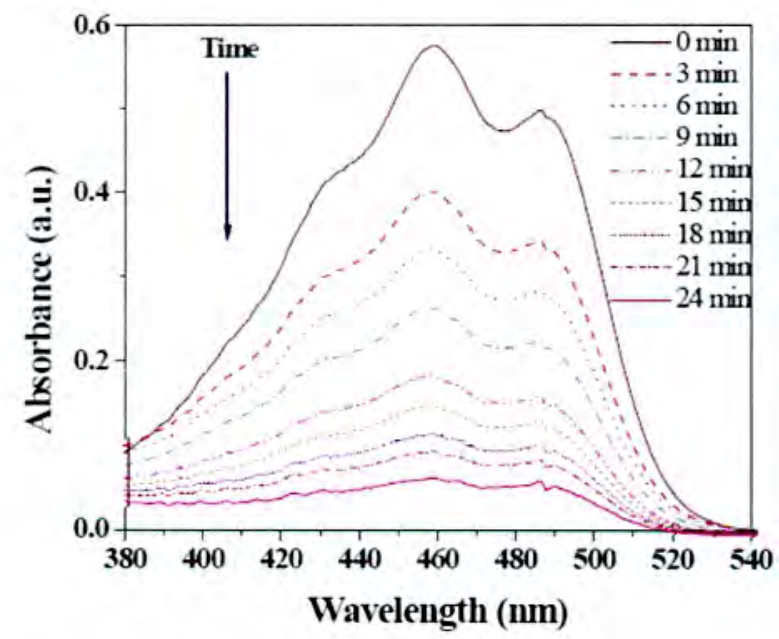

1 (b)

Figura 1. Espectros de Absorción de $\beta$-caroteno expuesta a irradiación UV a longitudes de onda de 366 nm (a) durante 48 minutos y $254 \mathrm{~nm}$ (b) durante 24 minutos. 
Tiburcio, J. \& Hernández, F. Estudio del proceso de fotodegradación de ß-caroteno en una solución de tetrahidrofurano (THF) usando espectroscopia de absorción óptica y fluorescencia.

tes excitados (Nielsen et al., 1996).

Para determinar el orden del proceso de fotodegradación de $\beta$-caroteno en THF bajo irradiación a $366 \mathrm{~nm}$ y $254 \mathrm{~nm}$, se graficaron la máxima absorbancia a una longitud de onda de $455 \mathrm{~nm}$ (A) vs. tiempo de irradiación (ver Figura 2). En la Figura 2 graficamos la función $\ln (\mathrm{A}) v$ s. $t$ y su correspondiente ajuste lineal. El modelo cinético usado corresponde a una reacción de primer orden en el reactante siguiendo la relación expresada por $\ln (A)=\ln \left(A_{0}\right)$-kt. En esta ecuación, A es proporcional a la concentración de $\beta$ carotene tal como se describe en la ley de Beer. En la ausencia de oxígeno, los carotenoides se degradan mediante procesos unimoleculares. Tal como ha sido discutido en la literatura, la degradación de los carotenoides ocurre por el rompimiento de unos de los enlaces centrales $\mathrm{C}-\mathrm{C}$ mediante estados excitados. Este mecanismo origina la producción de radicales (Nielsen et al., 1996) que pueden contribuir a la activación de especies oxidativas (Bezbradica et al., 2005). Los valores de las constantes asociadas con la fotodegradación fueron determinadas usando el mayor ajuste lineal de los datos experimentales. Los valores obtenidos para las constantes son:

$$
k_{366}=(0,0016 \pm 0,0001) \mathrm{min}^{-1} \text { y } k_{254}=(0,039 \pm 0,001) \mathrm{min}^{-1}
$$

Estos resultados demuestran claramente que la molécula de $\beta$-caroteno es muy sensible a la fotodegradación por luz ultravioleta de corta longitud de onda.

Para corroborar la fotodescomposición de $\beta$ caroteno, medimos el espectro de emisión de este compuesto a diferentes tiempos de irradiación para dos diferentes concentraciones, $3 \times 10^{-5} \mathrm{M}(\bullet)$ y $1,5 \times 10^{-6} \mathrm{M}(\boldsymbol{\bullet})$. Considerando que la fluorescencia de $\beta$-caroteno en solución es bajo, sin embargo, ha sido reportado que este parámetro puede ser medido usando una fuente de excitación de alta intensidad (Bachilo \& Gillbro, 1994) (Cherry et al., 1968). En ese sentido, usamos un láser diodo a $410 \mathrm{~nm}$ para realizar esta medida específica.

En la figura 3, mostramos la gráfica de la intensidad

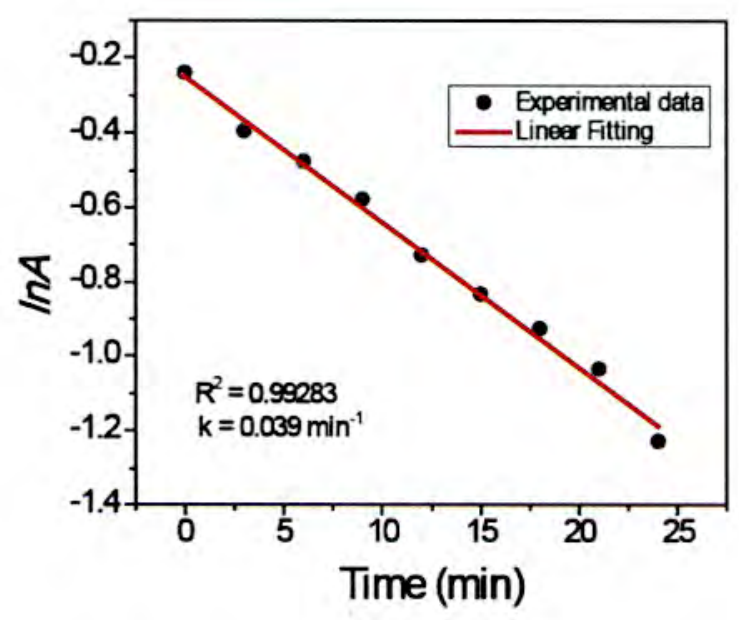

2(a) de emisión vs. tiempo para la solución, $\beta$-carotene/THF. La primera observación relevante es la inesperada elevación en la intensidad de fluorescencia de la solución altamente concentrada durante los primeros $30 \mathrm{~min}$ de irradiación, de experimentos previos, la irradiación de esta molécula a 254 $\mathrm{nm}$ induce una significante fotodescomposición, se podría esperar que ocurriria un decrecimiento en la intensidad de fluorescencia en el tiempo. Sin embargo, una observación de los resultados experimentales muestra que para una concentración umbral de $1,5 \times 10^{6} \mathrm{M}$ recién la fluorescencia muestra el comportamiento anticipado. Este resultado indica que para concentraciones de $\beta$-caroteno en THF superiores a $1,5 \times 10^{-6} \mathrm{M}$ ocurre una significante auto-absorción de la fluorescencia. Después de la fotodegradación de la molécula, la solución vuelve a manifestar un decrecimiento en la intensidad de fluorescencia tal como se muestra en la figura 3. Para comprobar este efecto se realizaron experimentos de fluorescencia para una solución de $\beta$-carotene/THF a una

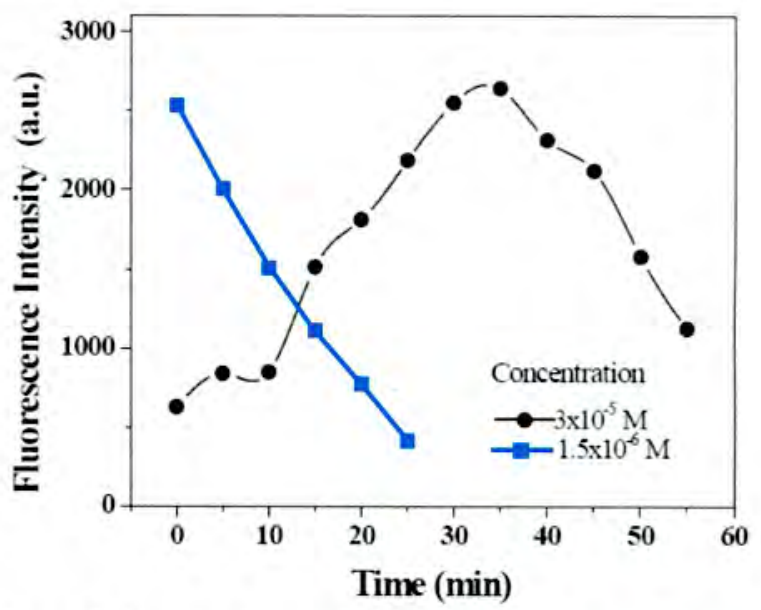

Figura 3. Ajuste de intensidad de emisión vs. tiempo, para $\beta$ caroteno en THF excitado con un diodo láser a $410 \mathrm{~nm}$ para diferentes tiempos de exposición a irradiación UV a $254 \mathrm{~nm}$.

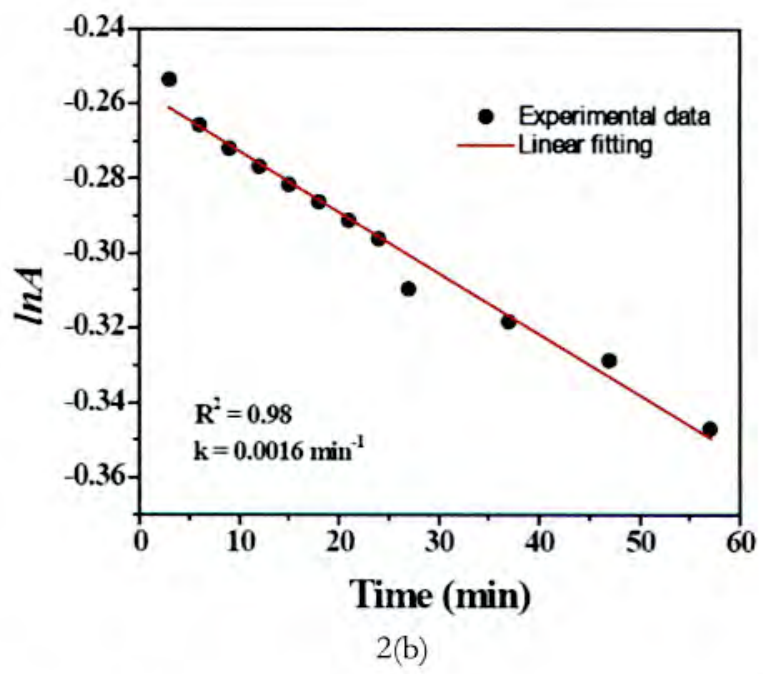

Figura 2. Ajuste lineal de datos experimentales para la absorbancia usando un modelo de cinética de primer orden para la fotodegradación de $\beta$-caroteno para (a) $366 \mathrm{nmy}$ (b) $254 \mathrm{~nm}$. 
Tïburcio, J. \& Hernández, F. Estudio del proceso de fotodegradación de $\beta$-caroteno en una solución de tetrahidrofurano (THF) usando espectroscopia de absorción óptica y fluorescencia.

concentración inicial de $1,5 \times 10^{-6} \mathrm{M}(\mathbf{\varpi})$.

Tal como se puede apreciar en la Figura 3, la intensidad de fluorescencia de la solución decrece a través del proceso de irradiación. Usando estos datos experimentales calculamos la constante de este proceso, considerando que la densidad de energía de la fuente de excitación usada en estos experimentos es casi 30 veces más intensa que la intensidad de la lámpara convencional a $254 \mathrm{~nm}$, es posible anticipar una descomposición fotoinducida ocasionada por el diodo laser.

Este efecto podría dar la impresión de una cinética más rápida y tal como se esperaba la constante calculada, $k_{25, F}=(0,045 \pm 0,002)$ minn $^{-1}$, para la fluorescencia es más grande que la obtenida mediante las medidas de la absorbancia.

\section{CONCLUSIONES}

El proceso de fotodegradación de $\beta$-caroteno en una solución de THF fue monitoreada exitosamente usando espectroscopia de absorción óptica y fluorescencia. Se determinó que el mecanismo de fotodegradación se ajusta a un modelo de cinética de primer orden. Estos resultados confirman la unimolecularidad del proceso de fotodescomposición de $\beta$-caroteno el cual toma lugar más rápidamente a longitudes de ondas cortas. Los experimentos de fluorescencia llevados a cabo confirmaron resultados previamente reportados en el sentido que para altas concentraciones se produce una auto-absorción de fluorescencia lo que podría llevar a incorrectas conclusiones. Los resultados de esta investigación representan una contribución a un mejor entendimiento de los procesos de fotodegradación de $\beta$ caroteno, los cuales podrian servir de ayuda para ser consideradas en potenciales usos de esta molécula, por ejemplo en la industria de alimentos y farmacéutica, las cuales trabajan con moléculas naturales antioxidantes.

\section{Agradecimientos}

Esta investigación fue parcialmente financiada por CONACYT México y a las facilidades otorgadas por la Universidad Nacional Jorge Basadre Grohmann de Tacna, Perú para la movilidad del autor de esta investigación.

\section{REFERENCIAS BIBLIOGRÁFICAS}

Allwood, M.C., Plane, J.H. (1984). The degradation of Vitamin A exposed to ultraviolet radiation. International journal of Pharmacentics, 19 207-213.

Bachilo S.M., Gillbro T. (1994). Beta-carotene S1 fluorescence, 5th International Conference on Laser Applications in Life Sciences, 2370 719-723.

Baranska, M., Schütze, W., Schulz, H. (2006). Determination of Lycopene and beta-Carotene Content in Tomato
Fruits and Related Products: Comparison of FTRaman, ATR-IR, and NIR Spectroscopy. Analytical Chemistry, 78 8456-8461.

Bezbradica, D., Milik-Askravic, J., Petrovi, S.D., SilerMarinkovic, S. (2005). An investigation of influence of solvent on the degradation kinetics of carotenoids in oil extracts of Calendula officinalis. Journal of the Serbian Chemical Society, 70 115-124.

Bonnie, T.Y.P., Choo, Y.M. (1999). Oxidation and thermal degradation of carotenoids. Journal of Oil Palm Research, 11 62-78.

Carnevale, J., Cole, E.R., Crank, G. (1979). Fluorescent Light Catalyzed Autoxidation of b-Carotene. Journal Agricultural and Food Chemistry, 27 462-463.

Carnevale, J., Cole, E.R., Crank, G. (1980). Photocatalyzed Oxidation of Paprika Pigments. Journal Agricultural Food and Chemistry, 28 953-956.

Cherry, R.J., Chapman, D., Langelaar, J. (1968). Fluorescence and Phosphorescence of beta-Carotene, Transactions of the Faraday Society, 64 2304-2307.

Gaziano, J.M., Manson, J.E., Buring, J.E., Hennekens, C.H. (1992). Dietary antioxidants and cardiovascular disease. Annals of the Nen York. Academy of Science, 669, 249-259.

Guan-Nan, Q., Shun-Li, O., Wei-Wei, W., Zuo-Wei, L., Cheng-Lin, S., Zhi-Wei, M. (2011). Influence of the ordered structure of short-chain polymer molecule all-trans-beta-carotene on Raman scattering cross section in liquid. Chinese Plyysics B, 2003037803.

Krisko, A., Piantanida, I., Kveder, M., Pifat, G. (2004). Analysis of beta-carotene absorbance for studying structural properties of human plasma low-density lipoproteins. Analytical Biochemistry. 331 177-182.

Nielsen, B.R., Mortensen, A., Jorgensen, K., Skibsted, L.H. (1996). Singlet versus Triplet Reactivity in Photodegradation of C40 Carotenoids. Journal Agricultural and Food Chemistry 1996, 44, 2106-2113, 442106-2113.

Siefermann-Harms, D. (1985). Carotenoids in photosynthesis. I. Location in photosynthetic membranes and light-harvesting function. Biochimica et Biophysica Acta (BBA) - Reviens on Bioenergetics, 811 325-355.

Sorg, O., Tran, C., Carraux, P., Didierjean, L., Saurat, J.-H. (1999). Retinol and Retinyl Ester Epidermal Pools Are Not Identically Sensitive to UVB Irradiation and Anti-Oxidant Protective Effect. Dermatology, 199 302-307.

Steinmetz, K.A., Potter, J.D. (1996). Vegetables, fruit, and cancer prevention: A review. Journal of the American Dietetic Association, $961027-1039$.

Vivas, M.G., Silva, D.L., Boni, L.D., Zalesny, R., Bartkowiak, W. (2011). Two-photon absorption spectra of carotenoids compounds. Journal of Applied Physics, 109 103529 .

\section{Correspondencia:}

José Antonio Tiburcio Moreno: jtiburc@hotmail.com
Fecha de Recepción: 29/04/2015

Fecha de Aceptación: 30/08/2015 